\title{
Frequencies Values of Orthotropic Composite Circular and Annular Plates
}

\author{
Kadir Mercan, Bekir Akgöz, Ciğdem Demir, Ömer Civalek* \\ Akdeniz University, Engineering Faculty, Civil Engineering Dept., Division of Mechanics \\ 07058, Antalya-TURKIYE \\ E-mail address: civalek@yahoo.com
}

Received date: April 2017

\begin{abstract}
Free vibration analysis of orthotropic composite annular plate is investigated. First-order shear deformation theory (FSDT) is used for equation of motion. Two different kernels such as Regularized Shannon delta (RSD) kernel and Lagrange delta sequence (LDS) kernel are used. The method of discrete singular convolution (DSC) is used for numerical simulation of governing equations to obtain the frequency values. It is shown that the convergence and accuracy of the DSC method is very good for vibration problem of orthotropic annular plate.
\end{abstract}

Keywords: Frequency, annular plate, discrete singular convolution, composite laminated.

\section{Introduction}

Free vibration analyses of shells and plates have widely studied by this time. Frequencies values of shell structures have major importance for their design in different fields. In literature, it is possible to find a few books on analysis and design of these structures [1-11]. Some important studies have been listed in references [9-42].

This paper is summarized in a few sections. In section 2, just main formulations for truncated conical shells and annular plates are given via Tong's [43] paper. The method of discrete singular convolution (DSC) is given in section 3. DSC solution for free vibration of orthotropic annular plates with is briefly defined in section 4. Results are listed in Section 5. Finally, a conclusion is located at the end of the paper.

\section{Fundamental Equations}

Geometry and parameters of conical shells and annular plates are depicted in Fig. 1. 


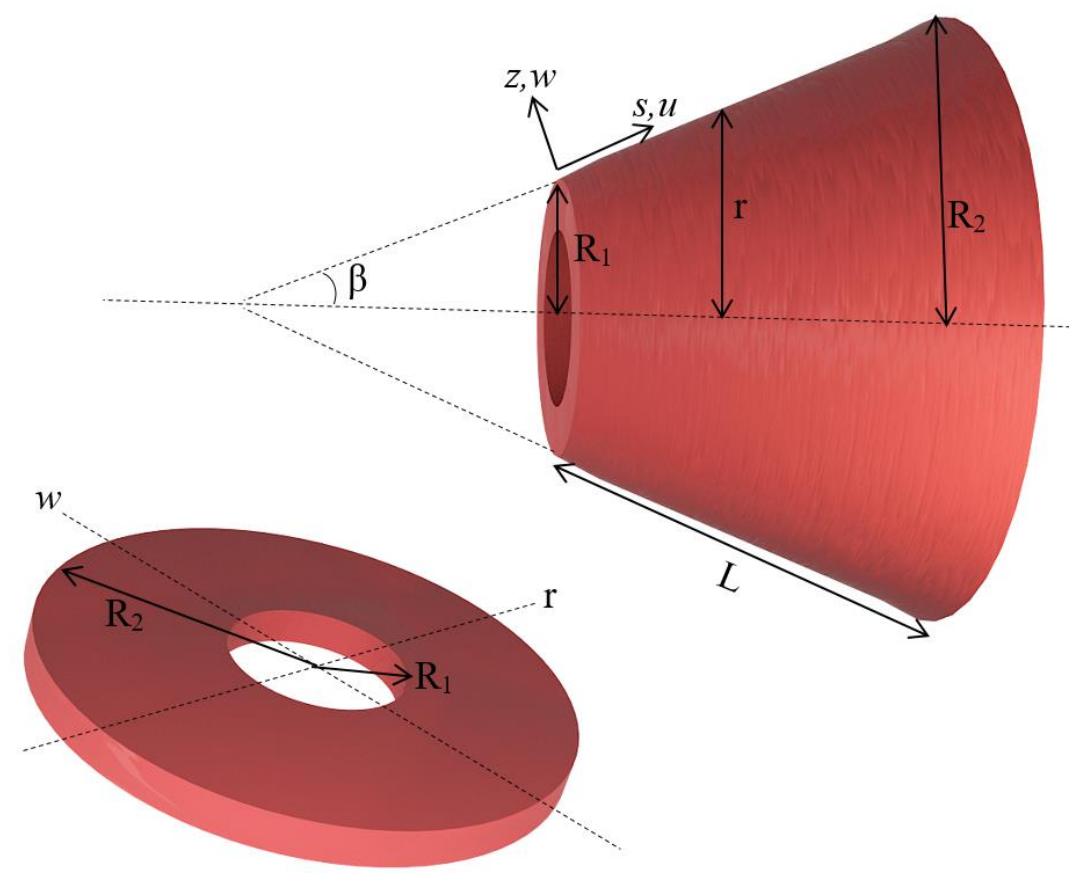

Fig. 1. Demonstration and notation of conical shell and annular plate

The equations of motion are [43]

$$
\begin{aligned}
\frac{\partial N_{x}}{\partial x}+ & \frac{1}{R(x)} \frac{\partial N_{x s}}{\partial s}+\frac{\sin \alpha}{R(x)}\left(N_{x}-N_{s}\right)=\rho h \frac{\partial^{2} u}{\partial t^{2}} \\
& \frac{\partial N_{x s}}{\partial x}+\frac{1}{R(x)} \frac{\partial N_{s}}{\partial s}+\frac{\cos \alpha}{R(x)} V_{s}+2 \frac{\sin \alpha}{R(x)} N_{x s}=\rho h \frac{\partial^{2} v}{\partial t^{2}} \\
\frac{\partial V_{x}}{\partial x}+ & \frac{\sin \alpha}{R(x)} V_{x}+\frac{1}{R(x)} \frac{\partial V_{s}}{\partial s}-\frac{\cos \alpha}{R(x)} N_{s}=\rho h \frac{\partial^{2} w}{\partial t^{2}} \\
& \frac{\partial V_{x}}{\partial x}+\frac{1}{R(x)} \frac{\partial V_{s}}{\partial s}-\frac{\cos \alpha}{R(x)} N_{s}+\frac{\sin \alpha}{R(x)} V_{x}=\frac{\rho h^{3}}{12} \frac{\partial^{2} \varphi_{x}}{\partial t^{2}} \\
\frac{\partial M_{x s}}{\partial x}+ & 2 M_{x s} \frac{\sin \alpha}{R(x)}+\frac{1}{R(x)} \frac{\partial M_{s}}{\partial s}-V_{s}=\frac{\rho h^{3}}{12} \frac{\partial^{2} \varphi_{s}}{\partial t^{2}}
\end{aligned}
$$

Moment and forces components can be defined as:

$$
\begin{aligned}
& \tilde{N}=\left\{\begin{array}{l}
N_{x} \\
N_{s} \\
N_{x s}
\end{array}\right\}=\int_{-h / 2}^{h / 2}\left\{\begin{array}{l}
\sigma_{x} \\
\sigma_{s} \\
\tau_{x s}
\end{array}\right\} d z \\
& \tilde{M}=\left\{\begin{array}{l}
M_{x} \\
M_{s} \\
M_{x s}
\end{array}\right\}=\int_{-h / 2}^{h / 2}\left\{\begin{array}{l}
\sigma_{x} \\
\sigma_{s} \\
\tau_{x s}
\end{array}\right\} z d z
\end{aligned}
$$




$$
\tilde{V}=\left\{\begin{array}{l}
V_{s} \\
V_{x}
\end{array}\right\}=\int_{-h / 2}^{h / 2}\left\{\begin{array}{c}
\tau_{s z} \\
\tau_{x z}
\end{array}\right\} d z
$$

For annular plates $(\alpha=90 ; \varphi=360)$ based on the FSDT the differential equations of motion can be defined in each direction:

$$
\begin{aligned}
& A_{11} \frac{\partial^{2} u}{\partial x^{2}}+\frac{A_{11}}{R(x)} \sin \alpha \frac{\partial u}{\partial x}-\frac{A_{22}}{R^{2}(x)} \sin ^{2} \alpha \cdot u+\frac{A_{33}}{R^{2}(x)} \frac{\partial^{2} u}{\partial s^{2}}+\frac{\left(A_{12}+A_{33}\right)}{R(x)} \frac{\partial^{2} v}{\partial x \partial s} \\
& -\frac{\left(A_{22}+A_{33}\right)}{R^{2}(x)} \sin \alpha \frac{\partial v}{\partial s}+\frac{A_{12}}{R(x)} \cos \alpha \frac{\partial w}{\partial x}-\frac{A_{22}}{R^{2}(x)} \sin \alpha \cdot \cos \alpha \cdot w-B_{11} \frac{\partial^{2} \varphi_{x}}{\partial x^{2}} \\
& +\frac{B_{11}}{R(x)} \sin \alpha \frac{\partial \varphi_{x}}{\partial x}-\frac{B_{22}}{R^{2}(x)} \sin ^{2} \alpha \cdot \varphi_{x}+\frac{B_{33}}{R^{2}(x)} \frac{\partial^{2} \varphi_{x}}{\partial s^{2}} \\
& +\frac{\left(B_{12}+B_{33}\right)}{R(x)} \frac{\partial^{2} \varphi_{s}}{\partial x \partial s}-\frac{\left(B_{22}+B_{33}\right)}{R^{2}(x)} \frac{\partial \varphi_{s}}{\partial s} \sin \alpha=\rho h \frac{\partial^{2} u}{\partial t^{2}} \\
& \frac{\left(A_{12}+A_{33}\right)}{R(x)} \frac{\partial^{2} u}{\partial x \partial s}+\frac{\left(A_{22}+A_{33}\right)}{R^{2}(x)} \sin \alpha \frac{\partial u}{\partial s}+A_{33} \frac{\partial^{2} v}{\partial x^{2}}+A_{33} \frac{\sin \alpha}{R(x)} \frac{\partial v}{\partial x} \\
& -\frac{A_{33}}{R^{2}(x)} \sin ^{2} \alpha \cdot v+\frac{A_{22}}{R^{2}(x)} \frac{\partial^{2} v}{\partial s^{2}}-\frac{A_{44}}{R^{2}(x)} \cos ^{2} \alpha \cdot v+\frac{\left(A_{22}+A_{44}\right)}{R^{2}(x)} \cos \alpha \frac{\partial w}{\partial s} \\
& +\frac{\left(B_{12}+B_{33}\right)}{R(x)} \frac{\partial^{2} \varphi_{x}}{\partial x \partial s}+\frac{\left(B_{22}+B_{33}\right)}{R^{2}(x)} \sin \alpha \frac{\partial \varphi_{x}}{\partial s}+B_{33} \frac{\partial^{2} \varphi_{s}}{\partial x^{2}}+B_{33} \frac{\sin \alpha}{R(x)} \frac{\partial \varphi_{s}}{\partial x} \\
& -\frac{B_{33}}{R^{2}(x)} \sin ^{2} \alpha \cdot \varphi_{s}+\frac{B_{22}}{R^{2}(x)} \frac{\partial^{2} \varphi_{s}}{\partial s^{2}}+A_{44} \frac{\cos \alpha}{R(x)} \cdot \varphi_{s}=\rho h \frac{\partial^{2} v}{\partial t^{2}} \\
& -\frac{A_{12}}{R(x)} \cos \alpha \frac{\partial u}{\partial x}-\frac{A_{22}}{R^{2}(x)} \cdot u \cdot \sin \alpha \cdot \cos \alpha-\frac{\left(A_{22}+A_{44}\right)}{R^{2}(x)} \cdot \cos \alpha \frac{\partial v}{\partial s}+A_{55} \frac{\partial^{2} w}{\partial x^{2}} \\
& +\frac{A_{55}}{R(x)} \sin \alpha \cdot \frac{\partial w}{\partial s}+\frac{A_{44}}{R^{2}(x)} \frac{\partial^{2} w}{\partial s^{2}}-\frac{A_{22}}{R^{2}(x)} \cdot w \cdot \cos ^{2} \alpha+A_{55} \frac{\partial \varphi_{x}}{\partial x}-\frac{B_{12}}{R(x)} \cos \alpha \cdot \frac{\partial \varphi_{x}}{\partial x} \\
& +\frac{A_{55}}{R(x)} \sin \alpha \cdot \varphi_{x}-\frac{B_{22}}{R^{2}(x)} \sin \alpha \cdot \cos \alpha \cdot \varphi_{x}+\frac{A_{44}}{R(x)} \cdot \frac{\partial \varphi_{s}}{\partial s} \\
& -\frac{B_{22}}{R^{2}(x)} \cdot \cos \alpha \frac{\partial \varphi_{s}}{\partial s}=\rho h \frac{\partial^{2} w}{\partial t^{2}} \\
& B_{11} \frac{\partial^{2} u}{\partial x^{2}}+\frac{B_{11}}{R(x)} \sin \alpha \frac{\partial u}{\partial x}-\frac{B_{22}}{R^{2}(x)} \cdot u \cdot \sin ^{2} \alpha+\frac{B_{33}}{R^{2}(x)} \frac{\partial^{2} u}{\partial s^{2}}+\frac{\left(B_{12}+B_{33}\right)}{R(x)} \frac{\partial^{2} v}{\partial x \partial s} \\
& -\frac{\left(B_{22}+B_{33}\right)}{R^{2}(x)} \sin \alpha \frac{\partial v}{\partial s}-A_{55} \frac{\partial w}{\partial x}+B_{12} \frac{\cos \alpha}{R(x)} \frac{\partial w}{\partial x}-\frac{B_{22}}{R^{2}(x)} \cdot w \cdot \sin \alpha \cos \alpha \\
& +D_{11} \frac{\partial^{2} \varphi_{x}}{\partial x^{2}}+D_{11} \frac{\sin \alpha}{R(x)} \frac{\partial \varphi_{x}}{\partial x}-\frac{D_{22}}{R^{2}(x)} \varphi_{x} \sin ^{2} \alpha+\frac{D_{33}}{R^{2}(x)} \frac{\partial^{2} \varphi_{x}}{\partial s^{2}}-A_{55} \varphi_{x} \\
& +\frac{\left(D_{12}+D_{33}\right)}{R(x)} \frac{\partial^{2} \varphi_{s}}{\partial x \partial s}-\frac{\left(D_{22}+D_{33}\right)}{R^{2}(x)} \frac{\partial \varphi_{s}}{\partial s} \sin \alpha=\rho h \frac{\partial^{2} \varphi_{x}}{\partial t^{2}}
\end{aligned}
$$




$$
\begin{gathered}
\frac{\left(B_{12}+B_{33}\right)}{R(x)} \frac{\partial^{2} u}{\partial x \partial s}+\frac{\left(B_{22}+B_{33}\right)}{R^{2}(x)} \frac{\partial u}{\partial s} \sin \alpha+B_{33} \frac{\partial^{2} v}{\partial x^{2}}+B_{33} \frac{\sin \alpha}{R(x)} \frac{\partial v}{\partial x} \\
-B_{33} \frac{\sin ^{2} \alpha}{R^{2}(x)} \cdot v+\frac{B_{22}}{R^{2}(x)} \frac{\partial^{2} v}{\partial s^{2}}+\frac{A_{44}}{R(x)} \cdot v \cdot \cos \alpha-\frac{A_{44}}{R(x)} \frac{\partial w}{\partial s}+\frac{B_{22}}{R^{2}(x)} \cos \alpha \frac{\partial w}{\partial s} \\
+\frac{\left(D_{12}+D_{33}\right)}{R(x)} \frac{\partial^{2} \varphi_{x}}{\partial x \partial s}+\frac{\left(D_{22}+D_{33}\right)}{R^{2}(x)} \sin \alpha \frac{\partial \varphi_{x}}{\partial s}-D_{33} \frac{\partial^{2} \varphi_{s}}{\partial x^{2}} \\
+D_{33} \frac{\sin \alpha}{R(x)} \frac{\partial \varphi_{s}}{\partial x}-\frac{D_{33}}{R^{2}(x)} \sin ^{2} \alpha \cdot \varphi_{s}+\frac{D_{22}}{R^{2}(x)} \frac{\partial^{2} \varphi_{s}}{\partial s^{2}}-A_{44} \cdot \varphi_{s}=\rho h \frac{\partial^{2} \varphi_{s}}{\partial t^{2}}
\end{gathered}
$$

\section{Discrete Singular Convolution (DSC)}

The method is originally introduced by Wei [44-47]. After the Wei's paper, the method of DSC have been used in many problems related to static, dynamic, free vibration and buckling analysis of structures [48-74]. A singular convolution $F$ can be formulated as [44]

$$
F(t)=(T * \eta)(t)=\int_{-\infty}^{\infty} T(t-x) \eta(x) d x
$$

In the study, regularized Shannon kernel (RSK) and Lagrange kernels are used.

Regularized Shannon kernel (RSK)

RSK kernel can be listed below [45-47]

$$
\delta_{\Delta, \sigma}\left(x-x_{k}\right)=\frac{\sin \left[(\pi / \Delta)\left(x-x_{k}\right)\right]}{(\pi / \Delta)\left(x-x_{k}\right)} \exp \left[-\frac{\left(x-x_{k}\right)^{2}}{2 \sigma^{2}}\right]_{; \sigma>0}
$$

Gaussian envelope is showed by symbol $\sigma$. In discrete form, any derivation can be written as

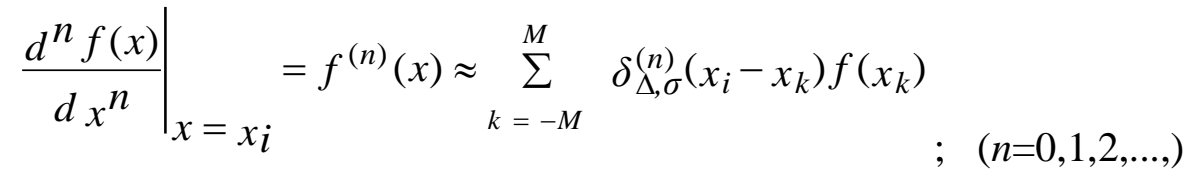

Lagrange delta sequence (LDS) kernel

LDS kernel is defined for $i=0,1, \ldots, N-1$ and $j=-M, \ldots, M$ is given by [44-50] 


$$
\mathfrak{R}_{i, j}(x)=\left\{\begin{array}{cc}
\prod_{k=i-M, k \neq i+j}^{i+M} \frac{x-x_{k}}{x_{i+j}-x_{k}}, & x_{i-M} \leq \mathrm{x} \leq x_{i+M}, \\
0 & \text { otherwise. }
\end{array}\right.
$$

In this case, the first and second order derivatives are given as

$$
\begin{aligned}
\delta_{\Delta, \sigma}^{(1)}(x)= & \sum_{i=-M ; i \neq k}^{M}\left(\frac{1}{x_{k}-x_{i}}\right) \prod_{i=-M, k \neq i}^{i+M} \frac{x-x_{i}}{x_{k}-x_{i}} \\
\delta_{\Delta, \sigma}^{(2)}(x)= & \sum_{\substack{i, m=-M ; i \neq k \\
m \neq k, i \neq m}}^{M}\left(\frac{1}{\left(x-x_{i}\right)\left(x-x_{m}\right)}\right) \prod_{\substack{i+M \\
i=-M, k \neq i}}^{i x-x_{i}} \frac{x}{x_{k}-x_{i}}
\end{aligned}
$$

\section{Results}

In this section, two examples are solved via two different kernels such as Regularized Shannon delta (RSD) kernel and Lagrange delta sequence (LDS). Frequency values for annular and circular plates have been obtained and results are listed in Tables 1-2 for orthotropic case. Results are obtained for clamped cases for annular and circular plates. Both kernels are useful for numerical discretization via DSC. It is shown that the $9^{*} 7$ grids are efficient for best convergence.

Table 1. Frequency values $\left(\Omega=\omega R_{1} \sqrt{\rho\left(1-v_{r} v_{\theta}\right) / E_{r}}\right.$ ) for orthotropic annular plate with C-C edges $\left(R_{1} / R_{2}=2 ; R_{1} / h=1000 ; \mathrm{E}_{\theta}=70 \mathrm{GPa}, \mathrm{v}_{\mathrm{c}}=0.3, \rho_{\mathrm{c}}=5700 \mathrm{~kg} / \mathrm{m}^{3}, \mathrm{E}_{\mathrm{r}}=1400 \mathrm{GPa}, \mathrm{v}_{\mathrm{r}}=0.3, \rho\right.$ $=7850 \mathrm{~kg} / \mathrm{m}^{3}$ )

\begin{tabular}{ccccc}
\hline Modes & \multicolumn{4}{c}{ Present DSC Results- RSD kernel } \\
\hline$(\sigma=2.8)$ & $7 \times 7(M=14)$ & $9 \times 7(M=14)$ & $9 \times 9(M=14)$ & $11 \times 9(M=14)$ \\
\hline 1 & 4.52420 & 4.52413 & 4.52413 & 4.52413 \\
2 & 4.74045 & 4.74038 & 4.74038 & 4.74038 \\
3 & 5.31453 & 5.31449 & 5.31442 & 5.31442 \\
4 & 6.10096 & 6.10090 & 6.10085 & 6.10085 \\
5 & 7.04989 & 7.04978 & 7.04976 & 7.04976 \\
\hline \multicolumn{5}{c}{ Present DSC Results- LDS kernel } \\
\hline$(\sigma=2.8)$ & $7 \times 7(M=14)$ & $9 \times 7(M=14)$ & $9 \times 9(M=14)$ & $11 \times 9(M=14)$ \\
\hline 1 & 4.52443 & 4.52438 & 4.52438 & 4.52438 \\
2 & 4.74059 & 4.74053 & 4.74051 & 4.74051 \\
3 & 5.31504 & 5.31493 & 5.31493 & 5.31493 \\
4 & 6.10103 & 6.10098 & 6.10094 & 6.10094 \\
5 & 7.05068 & 7.05016 & 7.05003 & 7.05003 \\
\hline
\end{tabular}


Table 2. Frequency values $\left(\Omega=\omega R_{1} \sqrt{\rho\left(1-v_{r} v_{\theta}\right) / E_{r}}\right.$ ) for orthotropic circular plate with clamped edges $\left(R_{1} / h=1000 ; \mathrm{E}_{\theta}=70 \mathrm{GPa}, v_{\mathrm{c}}=0.3, \rho_{\mathrm{c}}=5700 \mathrm{~kg} / \mathrm{m}^{3}, \mathrm{E}_{\mathrm{r}}=2800 \mathrm{GPa}, \mathrm{v}_{\mathrm{r}}=0.3\right.$, $\rho=7850 \mathrm{~kg} / \mathrm{m}^{3}$ )

\begin{tabular}{ccccc}
\hline Modes & \multicolumn{4}{c}{ Present DSC Results- RSD kernel } \\
\hline$(\sigma=2.8)$ & $9 \times 9(M=14)$ & $9 \times 7(M=14)$ & $11 \times 9(M=14)$ & $11 \times 11(M=14)$ \\
\hline 1 & 2.72081 & 2.72081 & 2.72081 & 2.72081 \\
2 & 3.37236 & 3.37236 & 3.37236 & 3.37236 \\
3 & 4.50756 & 4.50753 & 4.50753 & 4.50753 \\
4 & 4.98188 & 4.98182 & 4.98182 & 4.98182 \\
5 & 5.60235 & 5.60227 & 5.60227 & 5.60227 \\
$(\sigma=2.8)$ & $9 \times 9(M=14)$ & $9 \times 7(M=14)$ & $11 \times 9(M=14)$ & $11 \times 11(M=14)$ \\
\hline 1 & 2.72086 & 2.72086 & 2.72086 & 2.72086 \\
2 & 3.37244 & 3.37240 & 3.37240 & 3.37240 \\
3 & 4.50767 & 4.50760 & 4.50760 & 4.50760 \\
4 & 4.98195 & 4.98190 & 4.98188 & 4.98188 \\
5 & 5.60242 & 5.60236 & 5.60234 & 5.60234 \\
\hline
\end{tabular}

\section{Discussions}

In this paper discrete singular convolution method via FSDT shell theory is used for free vibration of annular and circular plates with orthotropic case. Two kernels namely Regularized Shannon delta (RSD) kernel and Lagrange delta sequence (LDS) kernel are used. The effects of grid numbers and kernel types on results have been investigated.

\section{Acknowledgements}

The financial support of the Scientific Research Projects Unit of Akdeniz University is gratefully acknowledged.

\section{References}

[1] Leissa, A.W., Vibration of plates, Acoustical Society of America, USA,1993.

[2] Reddy, J.N., Mechanics of laminated composite plates and shells: theory and analysis. (2nd ed.) New York: CRC Press, 2003.

[3] Qatu, M., Vibration of Laminated Shells and Plates, Academic Press, U.K., 2004.

[4] Soedel, W., Vibrations of shells and plates, Third Edition, CRC Press, 2004. 
[5] Shen, H.S., Functionally Graded Materials: Nonlinear Analysis of Plates and Shells, CRC Press, USA, 2009.

[6] Elishakoff, I., Demetris, P.D., Gentilini, C., Mechanics of Functionally Graded Material Structures, World Scientific, USA, 2016.

[7] Ugural, A.C., Stresses in Beams, Plates, and Shells, Third Edition, CRC Press, 2010.

[8] Ye, J., Laminated Composite Plates and Shells: 3D Modelling, Springer, 2003.

[9] Jin, G., Ye, T., Su, Z., Structural Vibration: A Uniform Accurate Solution for Laminated Beams, Plates and shells with general boundary conditions, Springer, 2015.

[10] Civalek, O., Finite Element analyses of plates and shells, Elazı̆̆g: Firat University, (in Turkish), 1998.

[11] Nie, G.J., Zhong, Z., Semi-analytical solution for three-dimensional vibration of functionally graded circular plates. Comput Methods Appl Mech Eng, 196, 4901-4910, 2007.

[12] Nie, G.J., Zhong, Z., Dynamic analysis of multidirectional functionally graded annular plates. Appl Math Model, 34, 608-616, 2010.

[13] Civalek, O., Gürses, M., Free vibration analysis of rotating cylindrical shells using discrete singular convolution technique. Int J Pres Ves Pip, 86(10), 677-683, 2009.

[14] Zhou, D., Lo, S.H., Cheung, Y.K., 3-D vibration analysis of annular sector plates using the Chebyshev-Ritz method. J Sound Vib, 320, 421 - 437, 2009.

[15] Civalek, O., Vibration analysis of laminated composite conical shells by the method of discrete singular convolution based on the shear deformation theory. Compos Part B Eng, 45(1), 1001-1009, 2013.

[16] Civalek, O., Free vibration analysis of composite conical shells using the discrete singular convolution algorithm. Steel Compos Struct, 6(4), 353-366, 2006.

[17] Civalek, O., The determination of frequencies of laminated conical shells via the discrete singular convolution method. J Mech Mater Struct, 1(1), 163-182, 2006.

[18] Zhao, X., Liew, K.M., Free vibration analysis of functionally graded conical shell panels by a meshless method, Compos Struct, 93, 649-664, 2011.

[19] Liew, K.M., Feng, Z.C., Vibration characteristics of conical shell panels with threedimensional flexibility. J Appl Mech, 67(2), 314-320, 2000.

[20] Liew, K.M., Ng, T.Y., Zhao, X., Free vibration analysis of conical shells via the element-free kp-Ritz method. J Sound Vib, 281, 627-645, 2005.

[21] Demir, Ç., Mercan, K., Civalek, O., Determination of critical buckling loads of isotropic, FGM and laminated truncated conical panel. Compos Part B: Eng, 94, 1-10, 2016.

[22] Tornabene, F., Fantuzzi, N., Viola, E., Ferreira, A.J.M., Radial basis function method applied to doubly-curved laminated composite shells and panels with a General Higherorder Equivalent Single Layer formulation. Compos Part B: Eng, 55, 642-659, 2013. 
[23] Liu, B., Ferreira, A.J.M., Xing, Y.P., Neves, A.M.A., Analysis of functionally graded sandwich and laminated shells using a layerwise theory and a differential quadrature finite element method. Compos Struct, 136, 546-553, 2016.

[24] Ferreira, A.J.M., Jorge, R.M.N., Roque, C.M.C., Free vibration analysis of symmetric laminated composite plates by FSDT and radial basis functions. Comput Method Appl Mech Eng, 194, 4265-4278, 2005.

[25] Ferreira, A.J.M., Roque, C.M.C., Neves, A.M.A., Jorge, R.M.N., Soares, C.M.M., Reddy, J.N., Buckling analysis of isotropic and laminated plates by radial basis functions according to a higher-order shear deformation theory. Thin-Wall Struct, 49(7), 804-811, 2011.

[26] Fantuzzi, N., Tornabene, F., Bacciocchi, M., Dimitri, R., Free vibration analysis of arbitrarily shaped Functionally Graded Carbon Nanotube-reinforced plates, Compos Part B: Eng doi.org/10.1016/j.compositesb.2016.09.021.

[27] Jin, G., Ma, X., Shi, S., Ye, T., Liu, Z., A modified Fourier series solution for vibration analysis of truncated conical shells with general boundary conditions. Appl Acoustics, 85, 82-96, 2014.

[28] Xie, X., Jin, G., Ye, T., Liu, Z., Free vibration analysis of functionally graded conical shells and annular plates using the Haar wavelet method. Appl Acoustics, 85, 130-142, 2014.

[29] Su, Z., Jin, G., Shi, S., Ye, T., Jia, Z., A unified solution for vibration analysis of functionally graded cylindrical, conical shells and annular plates with general boundary conditions. Int J Mech Sci, 80, 62-80, 2014.

[30] Jooybar, N., Malekzadeh, P., Fious, A., Vaghefi, M., Thermal effect on free vibration of functionally graded truncated conical shell panels. Thin Wall Struct, 103, 45-61, 2016.

[31] Malekzadeh, P., Heydarpour, Y., Free vibration analysis of rotating functionally graded cylindrical shells in thermal environment. Compos Struct, 94, 2971-81, 2012.

[32] Su, Z., Jin, G., Ye, T., Three-dimensional vibration analysis of thick functionally graded conical, cylindrical shell and annular plate structures with arbitrary elastic restraints. Compos Struct, 118, 432-447, 2014.

[33] Akgöz, B., Civalek O., Nonlinear vibration analysis of laminated plates resting on nonlinear two-parameters elastic foundations. Steel Compos Struct, 11(5), 403-421, 2011.

[34] Saidi, A.R., Baferani, A.H., Jomehzadeh, E., Benchmark solution for free vibration of functionally graded moderately thick annular sector plates. Acta Mech, 219, 309-335, 2011.

[35] Civalek, O., Ersoy, H., Free vibration and bending analysis of circular Mindlin plates using singular convolution method. Int J Num Method Biomed Eng, 25(8),907-922, 2009.

[36] Lin, C.C., Teng, C.S., Free vibration of polar orthotropic laminated circular and annular plates. J Sound Vib, 209, 797-810, 1988.

[37] Civalek, O., Dairesel Plakların Nöro-Fuzzy Tekniği ile Analizi. Dokuz Eylül Üniversitesi Mühendislik Fakültesi, 1(2), 13-31, 1999. 
[38] Wang, Q., Shi, D., Liang, Q., Ahad, F., An improved Fourier series solution for the dynamic analysis of laminated composite annular, circular, and sector plate with general boundary conditions. J Comp Mater, 50, 4199-4233, 2016.

[39] Civalek, O., Çatal, H.H., Linear static and vibration analysis of circular and annular plates by the harmonic differential quadrature (HDQ) method. Osmangazi Üniversitesi, Mühendislik ve Mimarlık Fakültesi Dergisi, 16(1), 43-71, 2003.

[40] Jin, G., Ma, X., Shi, S., Ye, T., Liu, Z., A modified Fourier series solution for vibration analysis of truncated conical shells with general boundary conditions. Appl Acoustics, 85, 82-96, 2014.

[41] Civalek, O., Çatal, H.H., Plakların Diferansiyel Quadrature Metodu ile Stabilite ve Titreşim Analizi, Teknik Dergi, 14(1), 2835-2852, 2003.

[42] Su, Z., Jin, G., Shi, S., Ye, T., Jia, X., A unified solution for vibration analysis Int J Mech Sci, 80, 62-80, 2014.

[43] Tong, L., Free vibration of laminated conical shells including transverse shear deformation. Int J Solids Struct, 31, 443-456, 1994.

[44] Wei, G.W., Solving quantum eigenvalue problems by discrete singular convolution. $J$ Phys B, 20, 343-352, 2000.

[45] Wei, G.W., A new algorithm for solving some mechanical problems. Comput Meth Appl Mech Eng, 190, 2017-2030, 2001.

[46] Wei, G.W., Vibration analysis by discrete singular convolution. J Sound Vib, 244, 535$553,2001$.

[47] Wei, G.W., Discrete singular convolution for beam analysis. Eng Struct, 23, 1045-1053, 2001.

[48] Hoffman, D.K., Wei, G.W., Zhang, D.S., Kouri, D.J., Shannon-Gabor wavelet distributed approximating functional, Chem Phys Letter, 287, 119-124, 1998.

[49] Shao, Z., Wei, G.W., Zhao, S., DSC time-domain solution of Maxwell's equations, $J$ Comput Phys, 189, 427-453, 2003.

[50] Wei, G.W., Zhao, Y.B., Xiang, Y., A novel approach for the analysis of high-frequency vibrations. J Sound Vib, 257, 207-246, 2002.

[51] Wei, G.W., Zhao, Y.B., Xiang, Y., Discrete singular convolution and its application to the analysis of plates with internal supports. Part 1: Theory and algorithm. Int J Num Meth Eng, 55, 913-946, 2002.

[52] Ng, C.H.W., Zhao, Y.B., Wei, G.W., Comparison of discrete singular convolution and generalized differential quadrature for the vibration analysis of rectangular plates. Comput Method Appl Mech Eng, 193, 2483-2506, 2004.

[53] Hou, Y., Wei, G.W., Xiang, Y., DSC-Ritz method for the free vibration analysis of Mindlin plates. Int J Num Meth Eng, 62, 262-288, 2005. 
[54] Civalek, O., Numerical analysis of free vibrations of laminated composite conical and cylindrical shells: discrete singular convolution (DSC) approach. J Comput Appl Math, 205, 251- 271, 2007.

[55] Civalek, O., Korkmaz, A., Demir, Ç., Discrete singular convolution approach for buckling analysis of rectangular Kirchhoff plates subjected to compressive loads on two opposite edges. Adv Eng Softw, 41, 557-560, 2010.

[56] Civalek, O., Analysis of thick rectangular plates with symmetric cross-ply laminates based on first-order shear deformation theory. J Compos Mater, 42, 2853-2867, 2008.

[57] Xin, L., Hu, Z., Free vibration of layered magneto-electro-elastic beams by SSDSC approach. Compos Struct, 125, 96-103, 2015.

[58] Xin, L., Hu, Z., Free vibration of simply supported and multilayered magnetoelectroelastic plates. Compos Struct, 121, 344-350, 2015.

[59] Baltacıŏlu, A.K., Civalek, Ö., Akgöz, B., Demir, F., Large deflection analysis of laminated composite plates resting on nonlinear elastic foundations by the method of discrete singular convolution. Int J Pres Ves Pip, 88, 290-300, 2011.

[60] Civalek, Ö., Akgöz, B., Vibration analysis of micro-scaled sector shaped graphene surrounded by an elastic matrix. Comp Mater Sci, 77, 295-303, 2013.

[61] Gürses, M., Civalek, Ö., Korkmaz, A., Ersoy, H., Free vibration analysis of symmetric laminated skew plates by discrete singular convolution technique based on first-order shear deformation theory. Int J Numer Methods Eng, 79, 290-313, 2009.

[62] Baltacioglu, A.K., Akgöz, B., Civalek, Ö., Nonlinear static response of laminated composite plates by discrete singular convolution method. Compos Struct, 93, 153-161, 2010 .

[63] Gürses, M., Akgöz, B., Civalek, Ö., Mathematical modeling of vibration problem of nano-sized annular sector plates using the nonlocal continuum theory via eight-node discrete singular convolution transformation. Appl Math Comput, 219, 3226-3240, 2012.

[64] Mercan, K., Civalek, Ö., DSC method for buckling analysis of boron nitride nanotube (BNNT) surrounded by an elastic matrix. Compos Struct, 143, 300-309, 2016.

[65] Xin, L., Hu, Z., Free vibration analysis of laminated cylindrical panels using discrete singular convolution. Compos Struct, 149, 362-368, 2016.

[66] Civalek, O., Mercan, K., Demir, C., Vibration analysis of FG cylindrical shells with power-law index using discrete singular convolution technique. Curved and Layer Struct, 3, 82-90, 2016.

[67] Civalek, O., Ulker, M., HDQ-FD integrated methodology for nonlinear static and dynamic response of doubly curved shallow shells. Struct Eng Mech, 19, 535-550, 2005.

[68] Yang, S.Y., Zhou, Y.C., Wei, G.W., Comparison of the discrete singular convolution algorithm and the Fourier pseudospectral method for solving partial differential equations. Comp Phys Commun, 143, 113-135, 2002. 
[69] Civalek, O. Nonlinear dynamic response of laminated plates resting on nonlinear elastic foundations by the discrete singular convolution-differential quadrature coupled approaches. Compos Part B: Eng, 50, 171-179, 2013.

[70] Mercan, K., Civalek, Ö., Buckling Analysis of Silicon Carbide Nanotubes (SiCNTs). Int J Eng Appl Sci, 8(2), 101-108, 2016.

[71] Mercan, K., Demir, Ç., Akgöz, B., Civalek, Ö., Coordinate Transformation for Sector and Annular Sector Shaped Graphene Sheets on Silicone Matrix. Int J Eng Appl Sci, 7(2), 56-73, 2015.

[72] Wang, X., Xu, S., Free vibration analysis of beams and rectangular plates with free edges by the discrete singular convolution. J Sound Vib, 329, 1780-1792, 2010.

[73] Wang, X., Wang, Y., Xu, S., DSC analysis of a simply supported anisotropic rectangular plate. Compos Struct, 94, 2576-2584, 2012.

[74] Duan, G., Wang, X., Jin, C., Free vibration analysis of circular thin plates with stepped thickness by the DSC element method. Thin Wall Struct, 85, 25-33, 2014. 